\title{
Microsensor arrays for breath analysis
}

\author{
M. Righettoni, Angiola Forleo ${ }^{2}$, L. Francioso ${ }^{2}$, Pietro Siciliano ${ }^{2}$ and Sotiris E. Pratsinis ${ }^{1}$ \\ ${ }^{1}$ Particle Technology Laboratory, ETH Zurich, CH-8092 Zurich, Switzerland \\ righettoni@ptl.mavt.ethz.ch \\ ${ }^{2}$ Institute for Microelectronics and Microsystems, IMM-CNR, IT 73100 Lecce, Italy
}

\begin{abstract}
:
Breath analysis, an innovative non-invasive diagnostic technique, bears the potential of drastically reducing the costs of medical diagnostics offering a simple alternative to standard blood analysis. Here, a flame spray pyrolysis (FSP) reactor was used for synthesis and direct deposition of nanostructured metal oxide $\left(\mathrm{MO}_{\mathrm{x}}\right)$ films onto microsensor substrates. These sensors were assembled in an array and tested simultaneously for different analytes requiring low power consumption to heat the sensor to the operating target temperature. The sensors had varying responses to the different analytes depending on sensing material (e.g. $\mathrm{SnO}_{2}, \mathrm{WO}_{3}, \mathrm{ZnO}$ ), facilitating improvements on the selectivity of specific analytes (e.g. acetone, methanol, isoprene).
\end{abstract}

Key words: metal oxide, flame spray pyrolysis, microsensors, selectivity.

\section{Introduction}

Human breath analysis is an emerging field of medical diagnostics that promises rapid, noninvasive monitoring and even detection of illnesses [1]. Fully integrated gas sensors made of nanoparticle films are a key technological development for advancing portability and performance of breath analysis devices [2]. The requirements for application of exhaled breath analysis in clinical routine are still challenging. Moreover, the development of small hand-held devices able to provide reliable and continuous real-time measurement of important breath markers such as acetone is desirable. Chemoresistive gas sensors made of nanostructured metal oxide semiconductors offer a promising alternative [2] to more sophisticated chemical detection devices such as gas chromatography (GC), proton-transfer-reaction mass spectrometry (PTR-MS) and selected ion flow tube mass spectrometry (SIFT-MS).These sensors have a lower limit of detection (LOD) in parts-per-billion concentrations to most reducing or oxidizing analytes[3] and can be miniaturized and integrated in gas microsensors [4] at low cost [3]. Additionally, lower power consumption can be achieved that is a key parameter in order to make these devices portable. A major shortcoming, however, is their poor selectivity to specific analyte in the presence of interfering gases. This problem has been resolved partly by careful materials engineering (e.g. control of crystal phases, addition of dopants), use of filters in the devices (e.g. to control the relative humidity) and the arrangement of several sensors in arrays (e.g. electronic noses). Recently, the application of electronic noses to detect diseases was proposed and investigated in more detail [5]. Some researchers have shown the ability of these devices to identify bacteria, infections, and other diseases [6].

\section{Experimental}

A flame spray pyrolysis (FSP) reactor was used in combination with a water-cooled substrate holder for synthesis and direct deposition of different $\mathrm{MO}_{\mathrm{x}}$ nanostructured films onto microsensors substrate array [7].Each sensor consisted of a $1.5 \times 1.5 \mathrm{~mm}$ substrate, with a platinum heater embedded having a $440 \mathrm{x}$ $440 \mu \mathrm{m}$ active area and requiring only about $500 \mathrm{~mW}$ to heat up at $350^{\circ} \mathrm{C}$. The flame-made \& directly deposited sensing films were mechanically stabilized by in situ annealing with a particle free flame [4]. Flame settings are described more in detail elsewhere [2].The crystal size and phase composition were characterized by X-ray diffraction (XRD). Prior to sensing tests, the sensors were kept in an oven at $500{ }^{\circ} \mathrm{C}$ for 5 hours at ambient pressure to stabilize the nanoparticle size and avoid further sintering during sensor measurements. For gas sensing tests, the devices were soldered onto a commercial TO-39 socket and were hosted in a test chamber. The microsensors were heated at different operating 
temperatures by supplying a given voltage to the heating element. The sensor responses towards gases were carried out by applying a constant voltage of $1 \mathrm{~V}$ between the sensor electrodes and by monitoring the current by means of an electrometer (Keithley 6517A) equipped with a multiplexer module. A PC via Labview National Instruments software controlled the entire process. The desired gas concentrations were obtained from certified cylinders by a mass flow controller (MKS mod. 647B) and of mass flow meters. A total flow of $100 \mathrm{sccm}$ was fixed during the measurements. The sensor response $(S)$ is:

$\mathrm{S}=\mathrm{R}_{\text {air }} / \mathrm{R}_{\text {analyte }}-1$

where $R_{\text {air }}$ is the film resistance in air at a given relative humidity $(\mathrm{RH})$ and $\mathrm{R}_{\text {analyte }}$ is that resistance at a given concentration of acetone or ethanol at that $\mathrm{RH}$. The morphology of the deposited sensing films was investigated by FIB Zeiss NVISION 40.

\section{Results and Discussion}

The gas detectors consisted of $\mathrm{MO}_{x}\left(\right.$ e.g. $\mathrm{SnO}_{2}$, $\mathrm{ZnO})$ directly deposited by flame spray pyrolysis (FSP) nanostructured films onto microsensors substrate[7]. The films were mechanically stabilized by in situ annealing with a particle free flame [4]resulting in a homogenous nanoparticulate porous layer (Figure 1). These results are similar to the one obtained by in situ annealing of $\mathrm{SnO}_{2}$ films [4]. This high porosity of the film facilitates the analyte and product transport through the sensitive layer so that analyte-induced resistance changes occur rapidly in the whole sensitive layer.

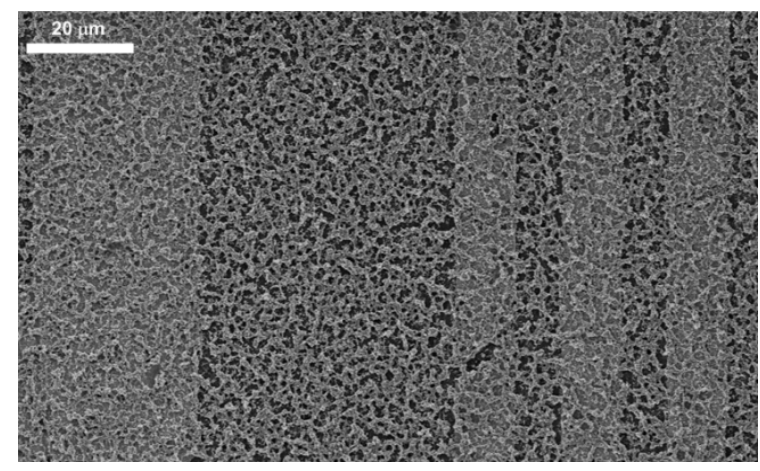

Fig. 1. Focused ion beam (FIB) image of $\mathrm{ZnO}$ nanoparticulate film onto a microsensor substrate deposited by FSP. The film is highly porous to allow rapid infiltration of analytes and evacuation of the reaction products facilitating short response \& recovery times.

These sensors were packaged and tested simultaneously in a Teflon chamber to different relevant breath markers such as acetone, a tracer to diabetes, or methanol, a tracer to renal problems. For example, ZnO-based sensors showed higher response to methanol than $\mathrm{SnO}_{2}$-ones (Figure 2). These sensor response variances could allow differentiation between gases by the use of different sensing materials in the sensor array enhancing the target analyte selectivity. Furthermore, pattern recognition methods, such as principal component analysis (PCA), could be used to analyze the gas mixture qualitatively and quantitatively.

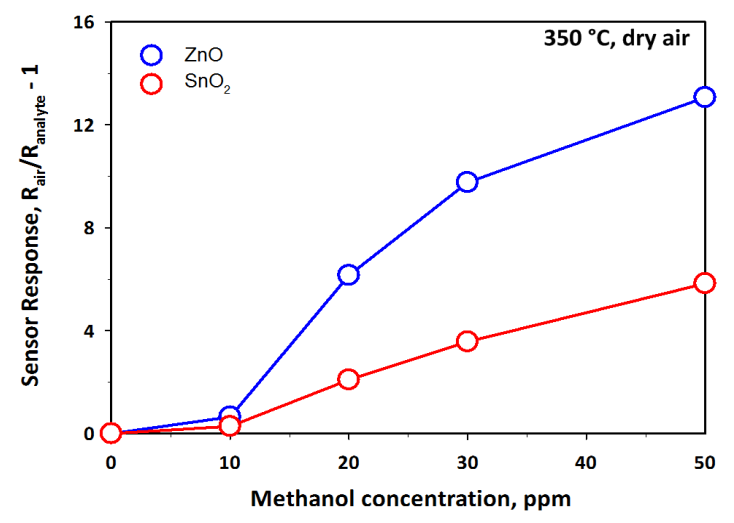

Fig. 2. Sensor response of $\mathrm{ZnO}$ (circles) and $\mathrm{SnO}_{2}$ (triangles) sensors to different $\mathrm{CH}_{3} \mathrm{OH}$

concentrations. The response of the $\mathrm{ZnO}$ sensor is more than double that of $\mathrm{SnO}_{2}$ at $50 \mathrm{ppm}$ methanol.

\section{Conclusions}

Flames spray pyrolysis has been used to directly deposit different $\mathrm{MO}_{\mathrm{x}}$ nanoparticles onto microsensor substrates. These sensors arrays showed a promising potential for improving the selectivity of chemo-resistive gas sensors.

\section{Acknowledgements}

This research was supported by the Swiss National Science Foundation, grant 200021_130582/1 and the European Research Council.

\section{References}

[1] W. Q. Cao, Y. X. Duan, Breath analysis: Potential for clinical diagnosis and exposure assessment, Clin. Chem. 52, 800-811 (2006).

[2] M. Righettoni, A. Tricoli, S. E. Pratsinis, $\mathrm{Si}: \mathrm{WO}_{3}$ Sensors for Highly Selective Detection of Acetone for Easy Diagnosis of Diabetes by Breath Analysis, Anal. Chem. 82, 3581-3587 (2010).

[3] G. Eranna, B. C. Joshi, D. P. Runthala, R. P. Gupta, Oxide materials for development of integrated gas sensors A comprehensive review, Crit. Rev. Solid State Mat. Sci. 29, 111-188 (2004).

[4] A. Tricoli, M. Graf, F. Mayer, S. Kuhne, A. Hierlemann, S. E. Pratsinis, Micropatterning layers by flame aerosol 
deposition-annealing, Adv. Mater. 20, 3005-3010 (2008).

[5] W. Ping, T. Yi, H. B. Xie, F. R. Shen, A novel method for diabetes diagnosis based on electronic nose, Biosens. Bioelectron. 12, 1031-1036 (1997).

[6] A. K. Pavlou, N. Magan, D. Sharp, J. Brown, H. Barr, A. P. F. Turner, An intelligent rapid odour recognition model in discrimination of Helicobacter pylori and other gastroesophageal isolates in vitro, Biosens. Bioelectron. 15, 333-342 (2000).

[7] L. Francioso, A. M. Taurino, A. Forleo, P. Siciliano, $\mathrm{TiO}_{2}$ nanowires array fabrication and gas sensing properties, Sens. Actuator B-Chem. 130, 70-76 (2008). 